\title{
Review of Optical Link Technology in Silicon Photonic Circuits
}

\author{
Chaitra $\mathbf{R}^{1}$, Divya $\mathbf{G}^{2}$, P Narashimaraja ${ }^{3}$ \\ ECE, R V College of Engineering, Bangalore, India
}

\begin{abstract}
Silicon photonics is the new advancement in the field of highly integrated devices on a single semiconductor substrate. The wide availability of fabrication facilities, high-density integration of photonic devices and the high probability of monolithic integration with electronic circuits offer the prospect for future reliable, low cost and energy-efficient. Silicon integrated chips have the capability to generate, modulate and analyse the light signals. Hence the have a varied application in computing, communications and sensing and optical interconnects. The application of photonics in optical interconnects is discussed in this paper. Here in we review recent progress in the engineering of new devices and important elements in silicon photonics, including low-loss waveguides, micro-ring resonators, modulators, photodetectors, transmitter and receiver architecture. We summarise the various types of optical ring resonators and coupling methods, in the paper. An attempt is also made to demonstrate the simulation of the single and double ring waveguides in LUMERICAL software.
\end{abstract}

Keywords: LUMERICAL, photonics, micro-ring resonators, photo-detectors, monolithic

\section{Introduction}

The tradeoffs faced by traditional electrical I/O can be overcome by using silicon-photonics technology .Silicon photonics is an emerging technology that has the potential to change the landscape of photonics. This technology offers compatibility with the existing CMOS technology and therefore provides many advantages such as high-volume, low-cost and manufacturing that is reliable with nanoscale precision. Applications of this technology can typically be found in telecommunication, data-communication, advanced instrumentation and sensing. The package cost and complexity would be reduced using this technology as it can be integrated with CMOS-based electronics allows for adding the driver and control electronics on the same chip. The addition of a photonic layer and interconnects would solve speed bottlenecks in future computing and chip platforms. Optical links demonstrate low loss at high symbol rates, have high distance insensitivity, and are immune to electromagnetic interference, making them a promising alternative Integrated silicon-photonics, in particular, holds promise of mass- produced photonics in low-cost silicon ICs . It also enables dense wavelength division multiplexing (DWDM ) where many data channels can pass through the same optic fibre cable, thereby increasing the bandwidth .

The benefits provided by optical fibre over electrical I/O are its absence of ground currents, its exceptionally low loss (covers larger distances between amplifiers), other power issues common to long parallel electric conductor runs (fibre optic has a dielectric nature and also depends on light rather than electricity for transmission), and its inherently high data-carrying capacity (Carries data using multiple modes) . Hence many electrical cables will be required to replace one optical link. Also, fibre optic cables do not experience any crosstalk unlike electrical cables .Therefore, Fibre optic cables can be installed in areas alongside power lines, utility lines, and railroad tracks or any other area having high electromagnetic interference (EMI).

Areas of high lightning-strike incidence can use non metallic all-dielectric cables. This paper introduces a technology for optical links between chips. It also provides a brief understanding about different ring resonators available and different coupling methods so as to choose the better one for photonic IC's.

\section{Photonic Optical Link}

The architecture of an DWDM chip-to-chip silicon-photonic link is shown in Fig. 1 [1]. A laser source, outside the chip produces a continuous light of $n$ wavelengths. The beam is collimated using a lens before being coupled to the single mode waveguide through a grating coupler. The transmit macro consists of resonant microrings, tuned to specific wavelengths. It modulates the data from the chip using an independent bitstream. This data exits the transmit chip using a second grating coupler into the receive macro of the second chip . The filter microrings tuned to each microring drop the light onto photodetectors to produce photocurrent, which the receivers resolve into data. 
International Journal of Science and Research (IJSR)

ISSN (Online): 2319-7064

Index Copernicus Value (2013): 6.14 | Impact Factor (2015): 6.391

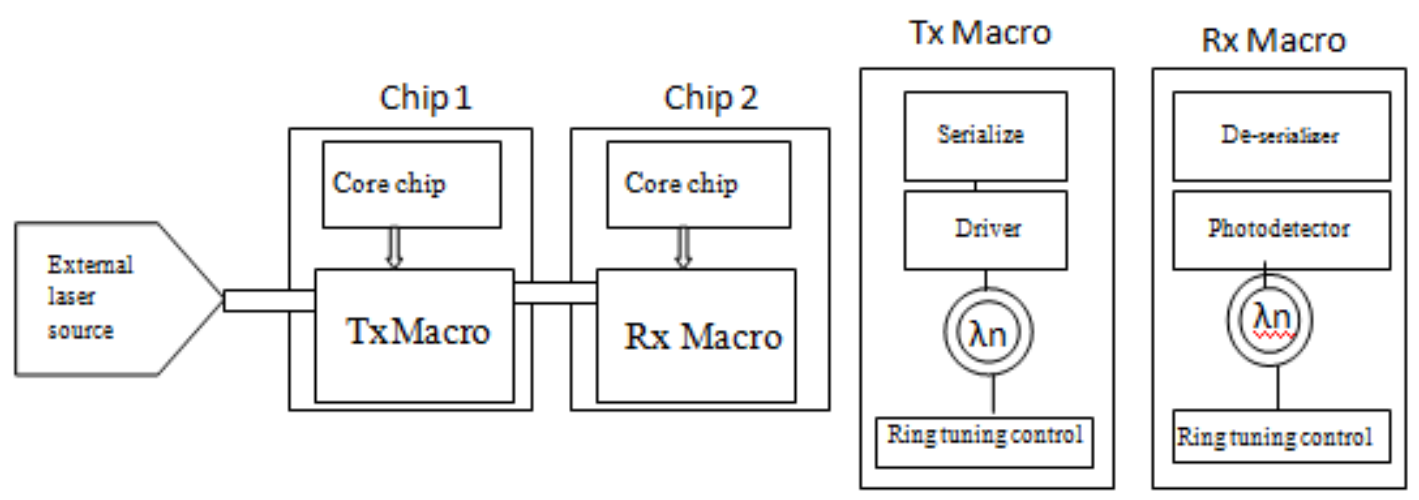

Figure 1: A chip-to-chip DWDM optical link using silicon-photonic

\section{Monolithic photonics platform in bulk}

Three modifications are made to the original CMOS process[1] to incorporate photonics in bulk CMOS. Due to the lack of Si layer, the first modification is the addition of a silicon implant amorphization step. This makes the waveguide less lossy. The second is the inclusion of a deeptrench isolation step (DTI), which creates an oxide filled trench beneath the waveguide that confines the laser beam within it. The third is a partial polysilicon etch (PPE) step. The PPE enables gratings to direct light in the right direction without the need for any reflectors to be embedded in the trench.

\section{Ring Resonator}

Ring resonators form the main component of the photonic circuit. The ring resonator is simply a transmission line formed in a closed loop. The basic circuit consists of the feed lines, coupling gaps and the resonator.

The concepts behind optical ring resonators is constructive interference, total internal reflection and optical coupling . When light of the resonant wavelength is passed through the loop from input waveguide, it builds up in intensity over multiple round-trips due to constructive interference and is output to the output bus waveguide which serves as a detector waveguide. Because only a select few wavelengths will be at resonance within the loop, the optical ring resonator functions as a filter. Additionally, as implied earlier, two or more ring waveguides can be coupled to each other to form an add/drop optical filter.

OOK (on -off keying) modulation technique is used in a ring resonator used to modulate the light. Changes in free carrier concentration are used to shift wavelength by changing the material's index of refraction. Carrier-injection modulators are $\mathrm{p}-\mathrm{i}-\mathrm{n}$ junctions that inject carriers into the intrinsic region during forward-bias, blue-shifting. Carrier-depletion modulators are $p-n$ junctions that deplete the carriers from the junction during reverse bias, red-shifting [2] . Forwardbiased operation of the junction also results in static power dissipation and poor energy-efficiency. Carrier --depletion designs avoid these issues.

\section{A. Operating Principle}

The ring resonator operates on 3 principles:
1. Total Internal Reflection [TIR]: When a ray of light strikes the boundary and fails to refract ,the phenomenon is known a TIR .If the angle of incidence is greater than the critical angle ,and the refractive index of the medium_2 is less than medium_1,the light reflects totally into medium 1.This techniques is used in ring resonator.

2. Interference: Interference causes two waves to superimpose to form a resultant wave with higher or lesser amplitude. As the light in the ring resonator completes multiple cycles around the ring component, it will interfere with the other light still in the loop. If there are no losses, due to absorption or imperfect coupling and resonance condition is met ,then the light emitted from the ring is same as the light incident on it.

3. Optical Coupling: A part of the beam of light passing through the waveguide gets coupled with the ring resonator. The coupling length, distance and the refractive indices between the waveguide and the optical ring resonator affects the coupling between the waveguide and the resonator. As the distance decreases, the coupling increases. The coupling length on the other hand has an inverse relationship with coupling. The refractive indices of the medium in between the waveguide and the resonator, ring resonator and the waveguide impacts the optical coupling.

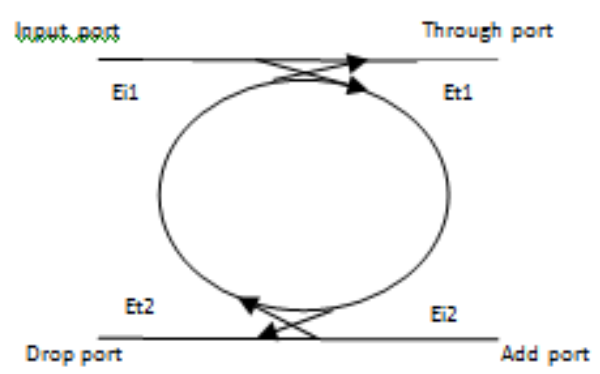

Figure 2: Single ring resonator

\section{B. Single ring waveguide}

For complete transfer to happen, the ring resonator should support 2 modes of operation. In case of single resonant mode of operation, it will decay through both waveguides along the forward and backward directions which introduces the reflection. 


\section{International Journal of Science and Research (IJSR) \\ ISSN (Online): 2319-7064}

Index Copernicus Value (2013): 6.14 | Impact Factor (2015): 6.391

The input light enters through the straight wave guide port 1 shown in Fig. 2 [6]. The light travels through the straight wave guide ,a part of is coupled to the ring resonator and outputs through the second wave guide port 3.The reflected output travels through port 4 . The wavelength which is resonant with the resonantor passes through the ring. For example, $\lambda i$ satisfies the resonant condition, that is,

$$
\text { neff } \mathrm{L}=\mathrm{m} \lambda \mathrm{i} \text {. }
$$

The coupling of the wave with wavelength $\lambda \mathrm{i}$ will be enhanced and all others will be suppressed. As a result, only $\lambda \mathrm{i}$ will be dropped from port 3 , while the rest of the wavelengths will pass through and output from Terminal 2. Here neff is the effective index of the bending waveguide, $\mathrm{L}$ is length of the ring, and $\mathrm{m}$ is an integer.

\section{Double ring resonator}

Double Ring Resonators (shown in Fig. 3) are coupled ring resonator devices consisting of two ring waveguides coupled to one or more bus waveguides. The double ring resonator consist of 2 rings either parallel or series coupled to the same wave guide. The rings are spaced apart and not coupled to one another. The space where the rings are close to the bus waveguide creates two coupling regions separated by a distance Ls along the bus waveguide.

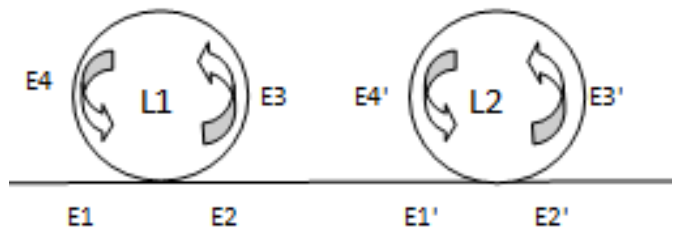

Figure 3: Double ring resonator

\section{Parallelly coupled resonator}

Light entering the bus waveguide shown in Fig. 4 is coupled to the first ring through evanescent coupling and travels along the circumference L1 of the ring before entering the same coupling region again. Transfer of power takes place at the coupling region. The light leaving the region travels a distance of Ls along the bus waveguide to enter the region where the second ring is coupled to the bus waveguide. Through evanescent coupling light enters the second ring, travels along its circumference L2 and re enters the coupling region.

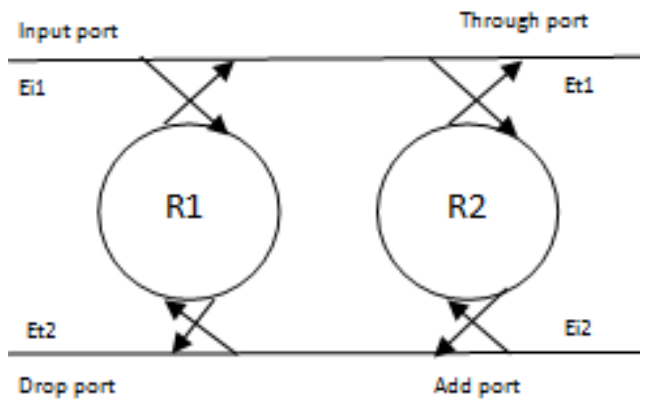

Figure 4: Parallel ring resonator without mutual ring coupling

\section{E. Serially coupled ring resonator}

In the serially coupled configuration shown in Fig. 5, each ring resonator is coupled to one another, and a signal that is to be dropped from the input port to the drop port must pass sequentially through each resonator. Because of this sequential power transfer, all resonators must be precisely resonant at a common wavelength. The resulting resonant line shape in the series configuration is determined physically by the separations between the ring resonators. In the parallel-coupled configuration, all resonators are Coupled to both the input and drop port waveguides, but usually not directly to one another (the resonators can also be coupled to one another resulting in a wavelength selective reflector). The resonators are instead indirectly coupled to each other by the optical path lengths along the input and output waveguides that interconnect them. These lengths determine the details of the resonant line shapes. An optical signal in the parallel configuration passes through all ring resonators simultaneously. This softens the requirement that the resonances of each ring have to be precisely identical. Nonaligned resonant frequencies instead lead to multiple peaks, or ripple in the line shape.

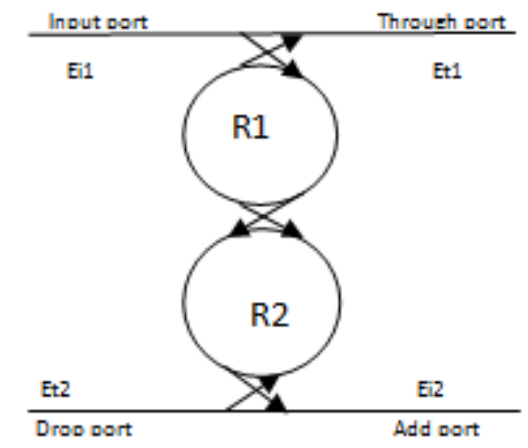

Figure 5: Serial double ring resonator

\section{F. Dumbbell ring resonator}

Dumbbell ring resonator shown in Fig. 6[3] ,contains three directional couplers and has the shape of a dumbbell .There is an additional directional coupler at the handle of the resonator. This coupler provides a link between the other two directional couplers unlike in double ring resonators, whose couplers are linked by vacuum. This linking in dumbbell resonators reduces leaking of data. The reflected signal consists of the travelling waves from various optical paths within the dumbbell shape structure. The reflection properties are studied using a transfer matrix . Field components of directional couplers

$$
E_{i}=\left[\begin{array}{c}
a_{i} \\
b_{i} \\
c_{i} \\
d_{i}
\end{array}\right], \quad i=1,2,3, \ldots, 6
$$

where ai and bi represent the field components for the forward travelling waves along the waveguide and ci and di represent the field components for the backward travelling waves. Coupling relationships for both the forward and backward travelling waves within the directional coupler using a $4 * 4$ coupling matrix: 


\section{International Journal of Science and Research (IJSR) \\ ISSN (Online): 2319-7064}

Index Copernicus Value (2013): 6.14 | Impact Factor (2015): 6.391

$$
C=\frac{1}{-j \kappa}\left[\begin{array}{cccc}
-t & 1 & 0 & 0 \\
-t^{2}-\kappa^{2} & t & 0 & 0 \\
0 & 0 & -t & 1 \\
0 & 0 & -t^{2}-\kappa^{2} & t
\end{array}\right]
$$

where $\kappa=|\kappa| e^{-(j \beta+\alpha / 2) L_{C}}$ and $t=|t| e^{-(j \beta+\alpha / 2) L_{C}}$

are the cross-coupling and the straight-through coupling coefficients, respectively, of the directional coupler, $\beta$ is the propagation constant, $\alpha$ is the propagation loss coefficient, and LC is the coupling length.

$\operatorname{Pm}(\mathrm{m}=1 ; 2 ;::: ; 5)$ is the propagation transfer matrices for light propagating through lengths of waveguides Lm between the directional couplers. The relationship between the left port and the right port of the device is given by:

$$
E_{1}=C_{12}\left(P_{2} C_{34}+P_{5}\right)\left(P_{3} C_{34}+P_{4}\right)^{-1} C_{56} E_{6}
$$

The performance of the dumbbell shaped resonator is determined by the cross-coupling coefficients, k12, k34, and $\mathrm{k} 56$. It affects the quality factor and free spectral range.

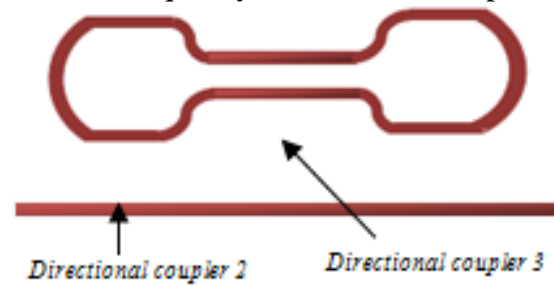

Figure 6: Dumbbell shaped resonator

\section{Types of Coupling}

The basic operation of any ring resonator its the coupling of the light wave to the ring. The coupling of the light depends on 3 factors [4]:

1) The distance,

2) The coupling length and

3) The refractive indices between the waveguide and the optical ring resonator.

When the mean circumference of the ring resonator is equal to an integral multiple of a guided wavelength, resonance is established. This may be expressed as:

$$
2 \cdot \pi \cdot r=n \cdot \lambda_{g} \quad \text { for } n=1,2,3, \ldots
$$

\section{A. Coupling gap:}

The gap between the straight waveguide and the ring, shown in Fig. 7 ,is known as coupling gap . These coupling gaps are modelled as capacitances depending on them they are categorised into different coupling methods .namely:

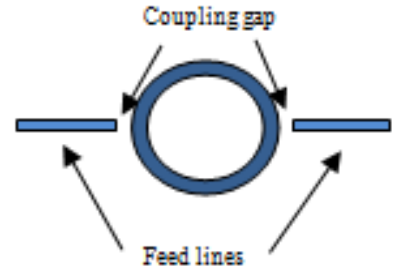

Figure 7: Microstrip ring resonator

\section{B. Loose Coupling}

In case of loose coupling the coupling gap is high and therefore the capacitance is negligible .Loose coupling is shown in Fig. 7. If the distance between the feed lines and resonator is kept large, then the capacitance is negligible and it does not affect the resonant frequencies. On the other hand, if the feed lines are moved closer to the resonator, the coupling increases .This is known as tight coupling .Though the coupling gap and capacitance is negligible, the increased coupling affects the resonant frequencies to deviate from the resonant frequency.

\section{Enhanced Coupling}

The feed lines are punched into the annular ring element as shown in the Fig 8 . There are 2 coupling gaps formed between each port and the feed lines. This type of coupling makes the resonant frequency less dependent on the coupling gap size.

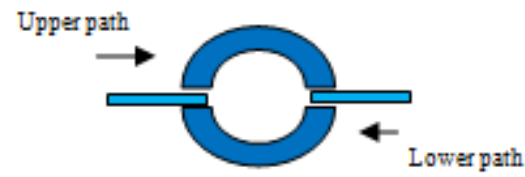

Figure 8: Enhanced coupling

\section{Matched stub coupling}

In the matched stubs coupling technique the gaps are formed between the feedlines and the matched stubs. The length of the stub affects the resonant frequency and the loss. The effect of stub length on loss is marginal but it greatly affects the resonant frequency. The advantage of matched-stub technique is you can keep the Q-factor constant when changing the stubs' length. The frequency change is significant hence this method is not suitable for ring resonator.The physical arrangement representing the matched stubs coupling technique is shown in Fig.9.

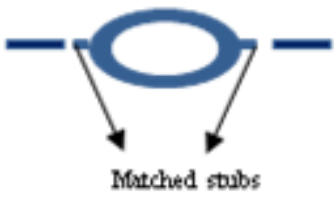

Figure 9: Matched stub coupling 
International Journal of Science and Research (IJSR)

ISSN (Online): 2319-7064

Index Copernicus Value (2013): 6.14 | Impact Factor (2015): 6.391

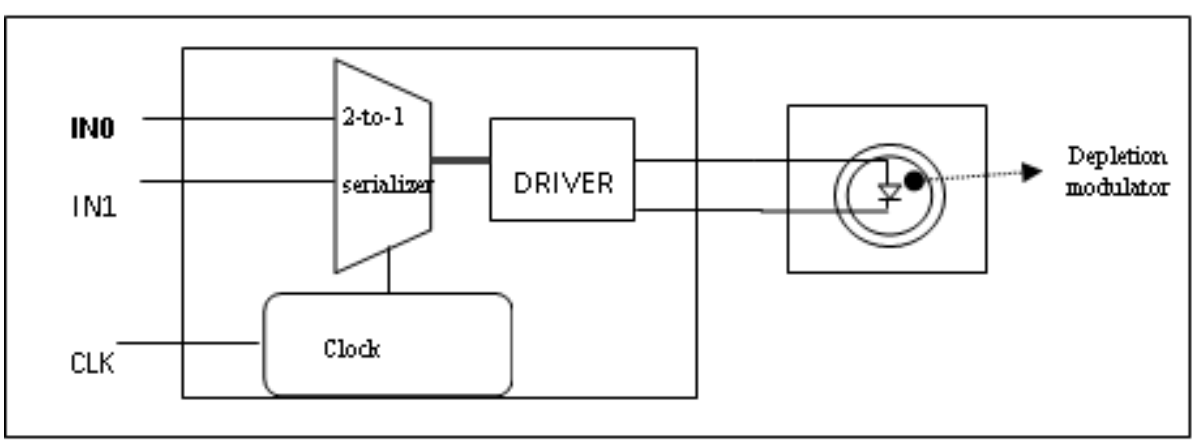

Figure 10: Depletion mode microring transmitter circuit

\section{Depletion-mode microring optical transmitter}

The transmitter block consists a serialiser to serialise the data. In order to provide a shift to the light depending on the data a push-pull driver circuit is implemented in the modulator. The red shift in the light is the 1s representation and blue shift is the 0 's representation. For logic 1, the transmitter applies a voltage of $-V D D$ to the modulator junction ,depleting the junction of carriers and for logic 0 , the circuit weakly forward biases the device to Vref-Vtn to enhance the junction providing a blue shift .The eye metric defines the difference for optical powers for

$$
\Delta T=\left|T_{1}-T_{0}\right|=\left|10^{-I L / 10}-10^{-(I L+E R) / 10}\right|
$$

modulated logic 1 and logic 0 levels. The normalized modulated input power is given by here $I L$ is the modulator insertion loss and $E R$ is the modulator extinction ratio, both given in $\mathrm{Db}$. The transmiiter circuit is shown in Fig. 10.

\section{Polysilicon based resonant optical receiver}

The receiver block consists of a defect detector and a receiver circuit as shown in Fig 11. Split diode technique is used to increase the speed of obtaining results at the receiver end. The PD microring is split into two isolated halves (PD0 and PD-1) whose outputs are given to two receiver halves, RX-0 and RX-1 respectively. Each receiver half gets half of the total photocurrent. The receiver half consists of a transimpedance amplifier whose transimpedance gain can be adjusted by changing the feedback resistor to convert the current into voltage, followed by a sense amplifier and finally a RS latch to store the results. Dummy PD's and TIA's are used to accommodate large dark current ranges, keeping the sense amplifier balanced for these large dark currents .

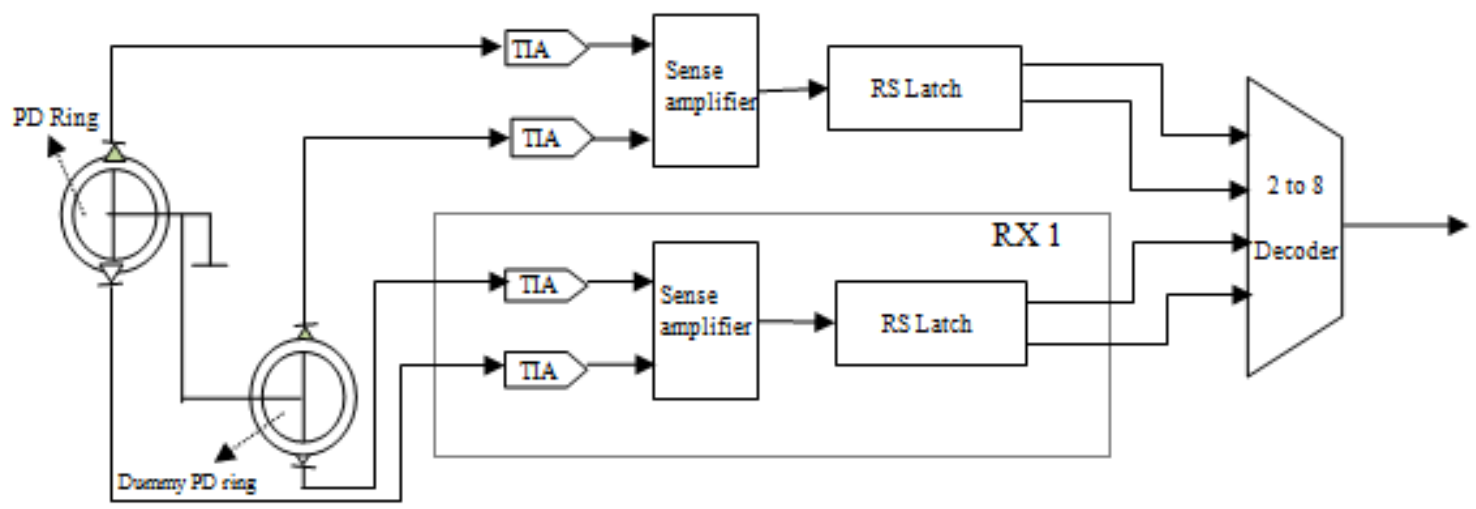

Figure 11: Schematic of receiver architecture

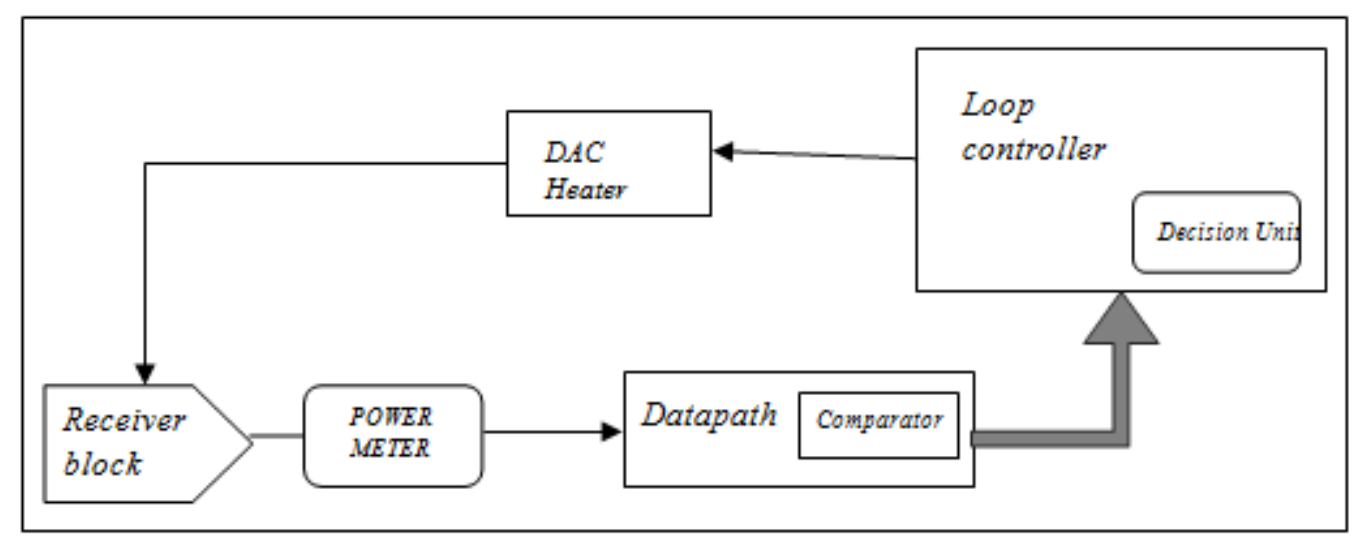

Figure 12: Schematic of wavelength locking system

Volume 5 Issue 6, June 2016 www.ijsr.net

Licensed Under Creative Commons Attribution CC BY 


\section{International Journal of Science and Research (IJSR) \\ ISSN (Online): 2319-7064}

Index Copernicus Value (2013): 6.14 | Impact Factor (2015): 6.391

\section{Ring resonator wavelength locking}

The wavelength locking circuit is a feedback circuit used to prevent any changes in the wavelength that each microring is tuned to , with any changes in temperature. The wavelength locking circuit is shown in Fig. 12 .The photocurrent level is tracked indirectly by a power meter to get the resonance back to the desired position. The values computed by the power meter is stored in the registers of the datapath and compared with the threshold value that is set in the look up table(LUT) of the controller. Then a decision is made to either increase or decrease the heating based on the comparison result. An 8 bit heater strength value generated as the output of the controller is then provided to the DAC heater circuit which controls the heating.

\section{Results}

Lumerical software was used for simulating the single and double ring. We started by breaking the circuit into different segments as shown in Fig. 13: and simulating each piece with a Straight Waveguide or Waveguide Coupler element from the Element Library (shown in Fig. 14).Also, Lumerical MODE solution was used to design and simulate a single ring resonator(Fig. 19).

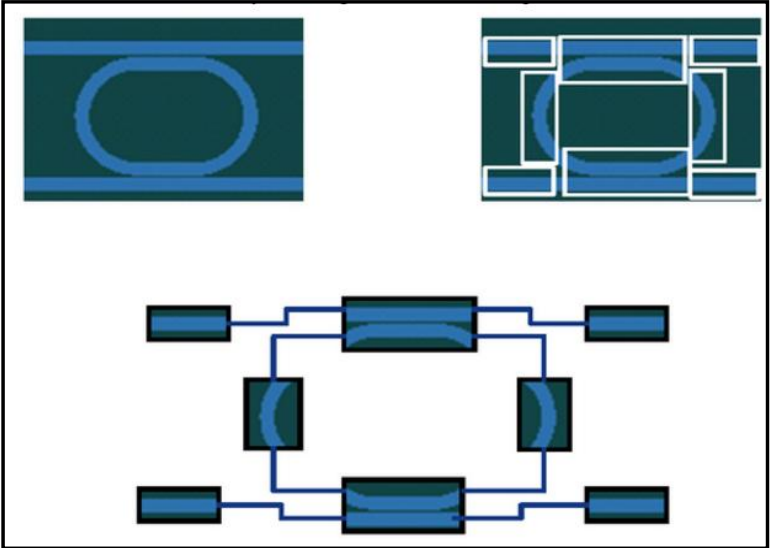

Figure 13: Sectional division of ring resonator

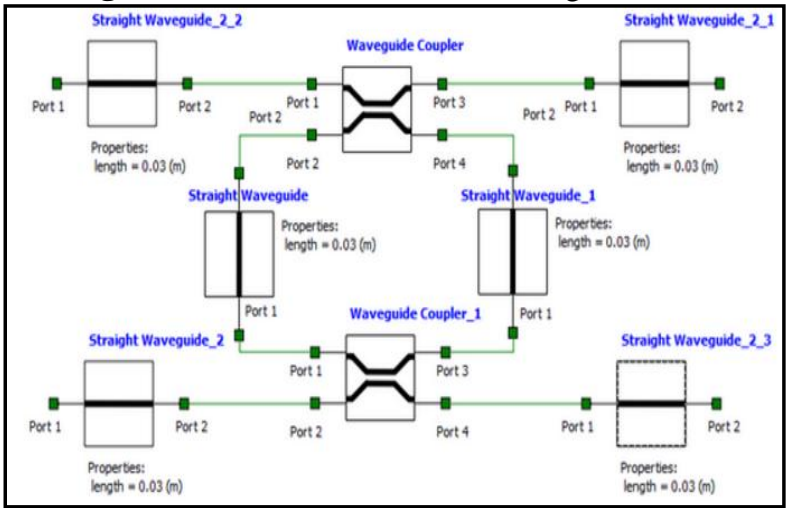

Figure 14: INTERCONNECT model of single ring resonator

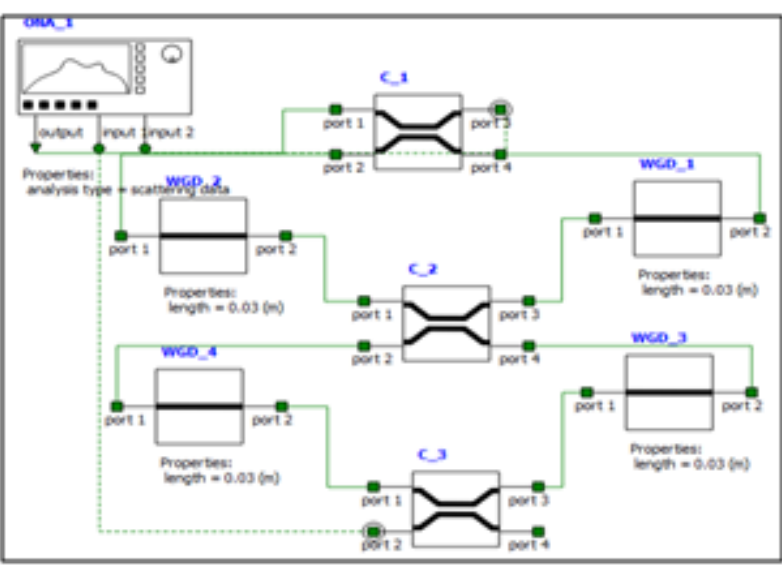

Figure 15: INTERCONNECT model for double ring resonator

Fig.15 was used to model a double ring resonator in Lumerical INTERCONNECT. The double ring resonator was initially, sectionally broken down into segments and each piece was simulated using elements from the library.

\section{Analysis}

\section{A.TE Transmission}

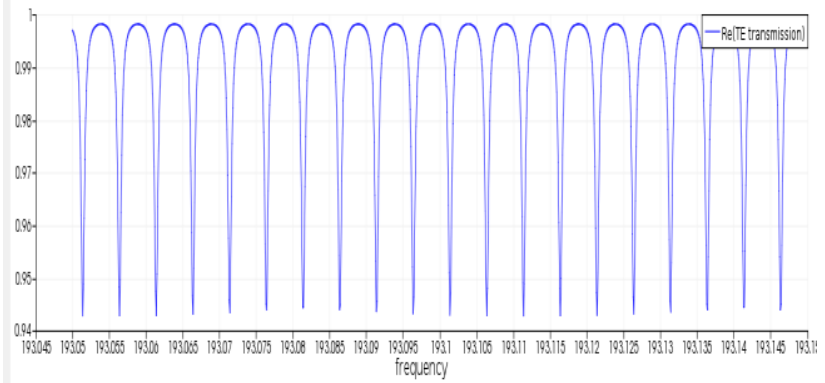

Figure 16: Frequency versus power plot

The frequency versus power plot is shown in Fig. 16. The wavelengths which are integral multiple of the diameter of the rings are dropped by the ring. These wavelength is known as the resonant frequency .The transmission coefficient at the resonant frequency is one (The transmission coefficient is the ratio of output power to the input power). For the other frequency the transmission coefficient drops down.The difference/Distance between any two lower peaks is known as the FSR of the resonator.

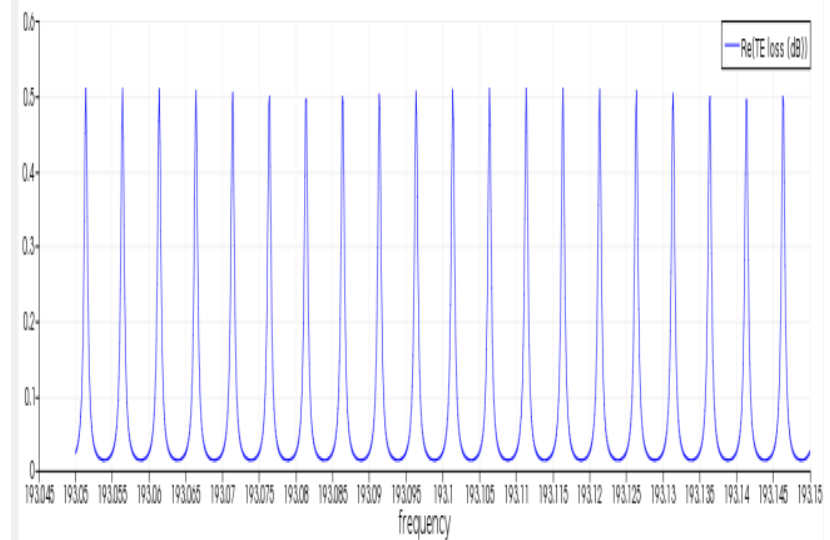

Figure 27: Loss plot for ring resonator 


\section{International Journal of Science and Research (IJSR) \\ ISSN (Online): 2319-7064 \\ Index Copernicus Value (2013): 6.14 | Impact Factor (2015): 6.391}

The Fig. 12 shows the plot of frequency versus loss. Due to the propagation and coupling distances ,there are lot of losses in the resonator. The thermal change will also effect the losses and hence changing the resonant frequency. Frequencies with minimum loss and with a constant loss should be chosen as resonant frequency.

\section{B. TE Gain}

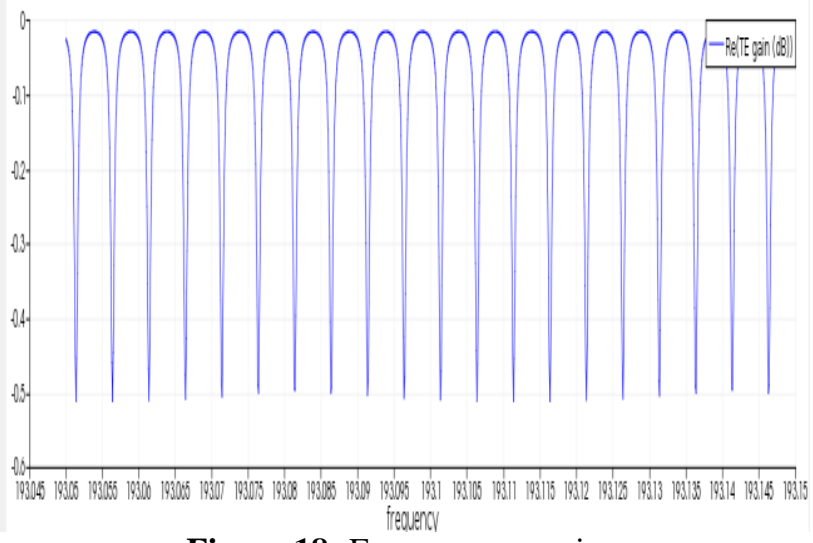

Figure 18: Frequency vs gain

The Fig. 18 gives a plot frequency versus gain. The maximum gain of the resonator is $0 \mathrm{db}$ at the resonance, the gain drops to negative $\mathrm{dB}$ in other regions.

\section{Lumerical mode simulation}

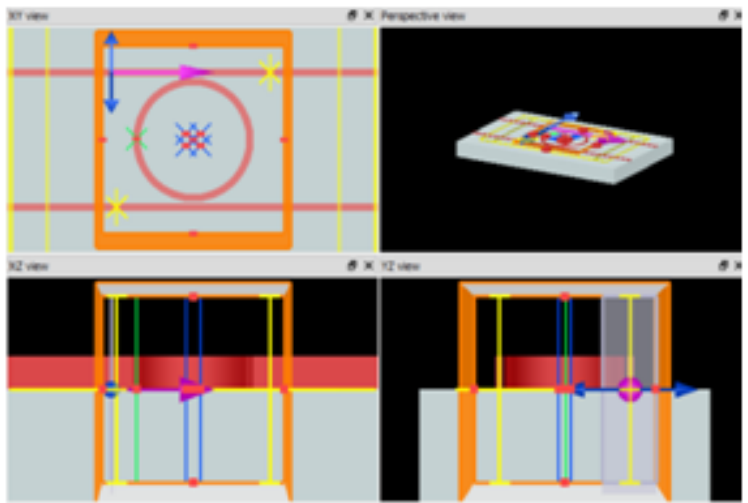

Figure 19: LUMERICAL MODE design of single ring resonator

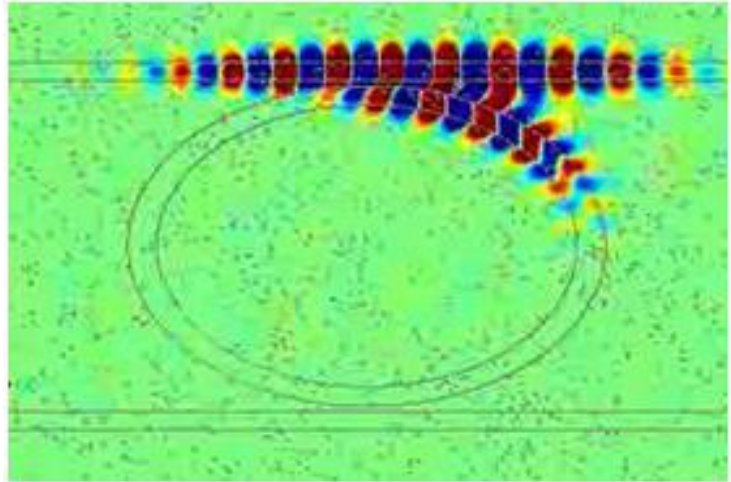

Figure 20: Coupling of the light wave with the ring resonator

The Fig.20 shows the coupling happening between the waveguide and the rings between the waveguides.

\section{Acknowledgment}

We would like to show our gratitude to Uma B.V, ECE HOD ,RVCE for sharing some pearls of wisdom with us during the course of the research. We are also immensely grateful to Ramavenkateswaran.N. , Assistant Professor for his comments on the earlier version of the manuscript, although any errors are our own and should not tarnish the reputation of these esteemed persons. We also thank our colleagues from R V College of Engineering and our parents who provided insights and expertise that greatly assisted our research.

\section{Conclusion}

The monolithic photonics platform in bulk CMOS was demonstrated in the paper. We have also comprehensively surveyed the various components of photonics including the photodetector, laser source, waveguide and the microring resonators. Photonics was integrated with minimal number of changes to the fabrication process. The ring resonators were detailed out along with the different coupling methods The transmitter, receiver architectures and the wavelength locking system were explained in the paper. The single and double ring resonator results and analysis , after simulation in LUMERICAL software are provided. Enabling the deployment of photonics in CMOS bulk process helps to further scale the optical link technology.

\section{References}

[1] Chen Sun, Michael Georgas, Jason Orcutt, Benjamin Moss, A Monolithically-Integrated Chip-to-Chip Optical Link in Bulk CMOS, IEEE journal of solid-state circuits, VOL. 50, NO. 4, APRIL 2015

[2] Wim Bogaerts, Pieter Dumon, Design Challenges in Silicon Photonics, IEEE journal of selected topics in quantum electronics, VOL. 20, NO. 4, JULY/AUGUST 2014

[3] Han Yun, Wei Shi, Xu Wang, Lukas Chrostowski, and Nicolas A. F. Jaeger, Dumbbell Micro-Ring Reflector ,Department of Electrical and Computer Engineering, University of British Columbia ,Canada

[4] George E. Chatzarakis, Review of different ring resonator coupling methods, School of pedagogical and technological education , January 2010

[5] G. Carpintero, K. Balakier, Z. Yang, R. C. Guzm’an, A. Corradi, A. Jimenez, G. Kervella, M. J. Fice, M. Lamponi, M. Chitoui, F. van Dijk, C. C. Renaud, A.Wonfor, E. A. J. M. Bente, R. V. Penty, I. H White, and A. J. Seeds, M,icrowave Photonic Integrated Circuits for Millimeter-Wave Wireless Communications, journal of lightwave technology, vol. 32, no. 20, october 15,2014

[6] Wim Bogaerts* , Peter De Heyn, Thomas Van Vaerenbergh, Katrien De Vos, Shankar Kumar Selvaraja, Tom Claes, Pieter Dumon, Peter Bienstman, Dries Van Thourhout, and Roel Baets

[7] Silicon ring resonators, Laser Photonics Rev. 6, No.1, 47-73 (2012) / DOI 10.1002/lpor.201100017 